\title{
Fontan fenestration closure and event-free survival
}

\author{
Bartlomiej R. Imielski, BS, Ronald K. Woods, MD, PhD, Kathleen A. Mussatto, PhD, RN, Yumei Cao, PhD, \\ Pippa M. Simpson, PhD, and James S. Tweddell, MD
}

Objectives: The purpose of the present study was to evaluate the association of open and closed Fontan fenestration status with event-free survival.

\begin{abstract}
Methods: All patients who underwent a fenestrated Fontan procedure at our institution from January 1994 through June 2007 were reviewed. Patient information was obtained from the medical records. The patients were assigned to 1 of 2 study groups, open or closed, according to their most recent fenestration status. Clinically relevant morbid events were tabulated, and Kaplan-Meier event analysis was used to create event-free probability curves with log-rank comparisons.
\end{abstract}

Results: A total of 161 patients were classified as open and 51 as closed. The median interval to an event was 1.1 years (interquartile range, 0.1-3.3 years) after the Fontan procedure. The median interval to closure was 1.2 years (interquartile range, 0.7-3.3 years). The median interval to an event was 1.5 years (interquartile range, 0.1-4.6 years) in the closed group and 1.1 years (interquartile range, 0.1-3.3 years) in the open group. Event-free probability analysis revealed no significant difference between the 2 groups $(P=.15)$. The median follow-up arterial oxygen saturation was greater in the closed group (96.0\%; interquartile range, $94.0 \%-97.0 \%)$ than in the open group $(91.0 \%$; interquartile range, $86.0 \%-95.0 \% ; P<.0001)$.

Conclusions: Fenestration closure was associated with greater arterial oxygen saturation but not greater eventfree survival. The interval to an event was slightly less than the interval to fenestration closure, suggesting potential merit in the evaluation of earlier fenestration closure. Adoption of specific fenestration management guidelines might help improve the overall outcomes and enhance the quality of future studies. ( $\mathrm{J}$ Thorac Cardiovasc Surg 2013;145:183-7)

Earn CME credits at

http://cme.ctsnetjournals.org

Fenestration of the Fontan circuit has been shown to improve early outcomes, including a decreased duration and quantity of chest tube drainage, a shorter duration of mechanical ventilation, and a shorter postoperative length of stay. ${ }^{1-5}$ Even in the present era in which fenestration is applied selectively by some groups, it continues to be used in high-risk patients, such as those with hypoplastic left heart syndrome.

Although a benefit in the early postoperative period, Fontan fenestration has theoretical long-term risks, including

\footnotetext{
From the Division of Cardiothoracic Surgery, Department of Surgery, Medical College of Wisconsin and Herma Heart Center, Children's Hospital of Wisconsin, Milwaukee, Wis.

This study was supported by an American Association for Thoracic Surgery Summer Intern Scholarship.

Disclosures: Authors have nothing to disclose with regard to commercial support. Read at the 38th Annual Meeting of The Western Thoracic Surgical Association, Maui, Hawaii, June 27-30, 2012.

Received for publication May 31, 2012; revisions received Aug 15, 2012; accepted for publication Sept 12, 2012; available ahead of print Oct 11, 2012.

Address for reprints: Ronald K. Woods, MD, PhD, FACS, FAAP, Division of Cardiothoracic Surgery, Department of Surgery, Medical College of Wisconsin, Children's Hospital of Wisconsin, 9000 West Wisconsin Ave, MS B730, Milwaukee, WI 53226 (E-mail: rwoods@chw.org).

$0022-5223 / \$ 36.00$

Copyright (c) 2013 by The American Association for Thoracic Surgery

http://dx.doi.org/10.1016/j.jtcvs.2012.09.006
}

cyanosis and systemic thromboembolic events due to a persistent right-to-left shunt combined with an increased risk of thrombus formation due to venous stasis and hypercoagulability. Alternatively, persistent fenestration might be a benefit because the lower central venous pressure might decrease the risk of exercise intolerance, protein-losing enteropathy (PLE), plastic bronchitis, and bradyarrhythmias. The question of whether and when to intentionally close a fenestration remains unanswered. Management protocols have tended to vary, ranging from active fenestration closure at predetermined intervals to a "hands-off" approach in which the natural history of fenestration status is allowed to progress. A cross-sectional study of a large cohort of Fontan patients by the Pediatric Heart Network found that $20 \%$ of patients had patent fenestrations at a median of 8.6 years after the Fontan procedure. ${ }^{6}$ Of those that were closed at follow-up, approximately $50 \%$ had been closed by active intervention (catheter based or surgical).

Therefore, we performed an analysis of a cohort of Fon$\tan$ patients to determine the association between fenestration status and morbid clinical events.

\footnotetext{
METHODS

Subjects

The records of all patients who had undergone a fenestrated Fontan procedure at Children's Hospital of Wisconsin from January 1994 through June 2007 were reviewed. The patients were assigned to 1 of 2 study
} 


\section{Abbreviations and Acronyms \\ $\mathrm{EFP}=$ event-free probability \\ $\mathrm{IQR}=$ interquartile range \\ PLE $=$ protein-losing enteropathy}

groups, open or closed, according to their most recent fenestration status as assessed by echocardiography. The presence of a detectable shunt by echocardiography, not the intervention history, determined the categorization as open or closed. Of a total of 218 patients, 6 were excluded because of either undifferentiated ventricular morphology (1 patient) or intraoperative conversion to a nonfenestrated status at the original Fontan procedure (5 patients).

The human research review board at the Children's Hospital of Wisconsin authorized the collection of data from the existing medical records and the waiver of the Health Insurance Portability and Accountability Act for the present retrospective study.

\section{Data Collection}

The study participants were identified from the Herma Heart Center Cardiology and Cardiothoracic Surgery database. Patient information was obtained from the medical records, echocardiograms, cardiac catheterization findings, and operative reports. De-identified patient information was maintained using the REDCap web-based research data capture application.

\section{Fenestrated Fontan Technique}

Fenestrations were routinely created in all patients undergoing the Fontan procedure. Earlier in the study interval, certain patients were selected for fenestration closure before leaving the operating room according to the hemodynamics and echocardiographic findings. For most patients in the present study (and routinely in the present era), the fenestrations were left open. Our technique of performing the lateral tunnel or extracardiac fenestrated Fontan procedure has been previously described. ${ }^{7}$ For all patients, a coronary punch was used to create a fenestration with a diameter of 3.5 to $4.0 \mathrm{~mm}$. For the lateral tunnel Fontan procedure, the fenestration was placed at the center of the Gore-Tex polytetrafluoroethylene baffle (WL Gore \& Associates, Flagstaff, Ariz). For the extracardiac Fontan procedure, the atriotomy that results from detaching the inferior vena cava from the right atrium was sewn to the Gore-Tex tube (WL Gore \& Associates) as a large circle to centrally encompass the fenestration and prevent the adjacent atrial wall from affecting the size of the fenestration. For the extracardiac Fontan procedure, a fenestration pursestring suture and snare (left open) were placed in an accessible subcutaneous pocket below the sternotomy to permit subsequent potential closure of the snare and fenestration.

\section{Fenestration Closure Technique}

Our general approach was to evaluate patients for fenestration closure at 1 to 3 years after the Fontan procedure. The patients were brought to the cardiac catheterization laboratory to first determine their suitability for closure. The decision to close the fenestration was individualized for each patient and was determined from a multidisciplinary assessment of test closure-induced changes in systemic venous pressure, cardiac output, and oxygen saturation. Closure was then completed during the same anesthetic session with echocardiographic and/or angiographic confirmation of the closure. For most patients undergoing closure, the fenestration snare was exposed through a limited skin incision, and the snare was closed and secured with hemoclips. A few patients, including those with residual shunts after attempted snare closure, underwent closure with an Amplatzer septal occluder (AGA Medical, Golden Valley, Minn).

\section{Outcome Variables}

Morbid events after Fontan palliation were defined as death, transplantation, Fontan takedown, New York Heart Association functional classification III or IV, pacemaker placement, PLE, stroke, thrombus, plastic bronchitis, subsequent thoracic surgery, or post-Fontan mechanical circulatory support. The interval during which a patient was free from an event determined the event-free survival. Events (but not patients) were excluded from the present analysis if they had occurred within 1 calendar day of the Fontan procedure. Such events were regarded as being more related to the overall impact of the surgical procedure and less to the fenestration. The follow-up duration was calculated from the date of the Fontan procedure to the last known clinical visit, if no events had occurred, or from the date of the Fontan procedure to the date of an event.

\section{Statistical Analysis}

Descriptive characteristics of the sample are summarized using the median and interquartile range, because the variables were skewed. The Wilcoxon-Mann-Whitney test was used to compare continuous variables and the chi-square test or Fisher exact test to compare fenestration groups (open vs closed) for patient characteristics. Kaplan-Meier survival analysis was used to assess the event-free survival, with Wilcoxon log-rank comparisons between the open and closed groups. All analyses were done in SAS, version 9.2 (SAS Institute, Cary, NC).

\section{RESULTS}

\section{Group Characteristics}

Of the 212 patients in the present cohort, 161 were categorized as open and 51 as closed. Included in the open group were 10 patients who had undergone fenestration closure interventions with subsequent echocardiographically determined residual shunts and 19 patients who had undergone fenestration closure on the same day as their last known clinical follow-up visit. Included in the closed group were 18 patients with spontaneously closed fenestrations. The patient characteristics are summarized in Table 1. No significant differences were found in the patient characteristics between the open and closed groups.

The mean and median duration of follow-up after the Fontan procedure was 3.8 and 3.5 years (interquartile range [IQR], 0.9-6.1 years) for the entire cohort, 3.1 and 2.4 years (IQR, 0.7-4.9 years) for the open group, and 5.9 and 6.0 years (IQR, 4.4-8.0 years) for the closed group, respectively. The mean and median interval from Fontan to fenestration closure was 2.1 and 1.2 years (IQR, 0.7-3.3 years), respectively.

\section{Events and Event-Free Probability}

Of a total of 92 events, 72 occurred in the open group (50 of 161 patients, $31 \%$ ) and 20 in the closed group (15 of 51 patients, $29 \%$ ). In the closed group, 7 patients had events before fenestration closure and 9 after fenestration closure. The events are summarized according to closure status in Table 2. The most notable differences between the open and closed groups occurred with the event of New York Heart Association class III or IV, which occurred in 16.8\% of the open compared with $2.0 \%$ of the closed group; and the event of PLE, which occurred in $4.3 \%$ of the open group compared with $0 \%$ in the closed group. 
TABLE 1. Patient characteristics

\begin{tabular}{|c|c|c|c|}
\hline Characteristic & Open & Closed & $P$ value \\
\hline Gender & & & .11 \\
\hline Male & $111(68.9)$ & $29(56.9)$ & \\
\hline Female & $50(31.1)$ & $22(43.1)$ & \\
\hline Diagnosis category & & & .84 \\
\hline Hypoplastic left heart syndrome & $76(47.2)$ & $21(41.2)$ & \\
\hline Double inlet left ventricle & $28(17.4)$ & $12(23.5)$ & \\
\hline Tricuspid atresia & $19(11.8)$ & $8(15.7)$ & \\
\hline Unbalanced atrioventricular canal & $10(6.2)$ & $4(7.8)$ & \\
\hline Heterotaxy & $11(6.8)$ & $2(3.9)$ & \\
\hline $\begin{array}{l}\text { Pulmonary atresia with intact } \\
\text { ventricular septum }\end{array}$ & $6(3.7)$ & $2(3.9)$ & \\
\hline Other & $11(6.8)$ & $2(3.9)$ & \\
\hline Ventricular morphology & & & .21 \\
\hline Left & $60(37.3)$ & $24(47.1)$ & \\
\hline Right & $101(62.7)$ & $27(52.9)$ & \\
\hline Fontan type & & & .94 \\
\hline Lateral tunnel & $37(23.0)$ & $12(23.5)$ & \\
\hline Extracardiac & $124(77.0)$ & $39(76.5)$ & \\
\hline Age at Fontan (y) & & & .80 \\
\hline Median & 2.92 & 3.05 & \\
\hline IQR & $2.31-3.72$ & $2.22-3.72$ & \\
\hline Weight at Fontan (kg) & & & .49 \\
\hline Median & 13.0 & 12.8 & \\
\hline IQR & $11.7-14.7$ & $11.0-14.9$ & \\
\hline
\end{tabular}

Data presented as $\mathrm{n}(\%)$ or median and IQR. IQR, Interquartile range.

In the event-free probability (EFP) analysis, the median interval to an event was 1.1 years (IQR, 0.1-3.3 years) for the open group and 1.5 years (IQR, 0.1-4.6 years) for the closed group. Figure 1 shows the EFP curves for the closed and open groups. The closed group demonstrated a marginally greater EFP; however, the difference was not statistically significant $(P=.08)$.

EFP calculated with intervals referenced to the time of Fontan has the benefit of a longer period of follow-up. Because the present analysis included events occurring before fenestration closure in the group ultimately undergoing fenestration closure, it could not exclusively evaluate the association of closed fenestration status with events. In an effort to address this issue, an additional analysis was completed, in which the median interval to fenestration closure was selected as the reference point for measuring the interval to an event. Therefore, the patients who had no follow-up subsequent to 424 days after the Fontan procedure were excluded, and events (but not patients) in both the open and the closed groups occurring before 424 days after the Fontan procedure were excluded. For the latter analysis, 47 patients were in the closed group and 102 patients were in the open group. Figure 2 shows the EFP curves for the open and closed groups, with no significant difference found between the 2 groups $(P=.15)$. For this comparison, the median length of follow-up was 2.8 years (IQR, 1.6-5.1 years) for the
TABLE 2. Event characteristics stratified by fenestration group

\begin{tabular}{lclcc} 
& & \multicolumn{2}{c}{ Closed } \\
\cline { 3 - 4 } \multicolumn{1}{c}{ Event type } & Open & $\begin{array}{c}\text { Before } \\
\text { closure }\end{array}$ & $\begin{array}{c}\text { After } \\
\text { closure }\end{array}$ & Cumulative \\
\hline Death & $5(3.1)$ & $0(0)$ & $3(5.9)$ & $8(3.8)$ \\
Heart transplantation & $1(0.6)$ & $0(0)$ & $0(0)$ & $1(0.5)$ \\
Fontan takedown & $1(0.6)$ & $0(0)$ & $0(0)$ & $1(0.5)$ \\
NYHA III or IV* & $27(16.8)$ & $0(0)$ & $1(2.0)$ & $28(13.2)$ \\
Pacemaker & $7(4.3)$ & $1(2.0)$ & $1(2.0)$ & $9(4.2)$ \\
PLE & $7(4.3)$ & $0(0)$ & $0(0)$ & $7(3.3)$ \\
Stroke & $3(1.9)$ & $0(0)$ & $0(0)$ & $3(1.4)$ \\
Thrombus & $9(5.6)$ & $1(2.0)$ & $3(5.9)$ & $13(6.1)$ \\
Plastic bronchitis & $0(0)$ & $1(2.0)$ & $0(0)$ & $1(0.5)$ \\
Subsequent thoracic surgery & $11(6.8)$ & $5(9.8)$ & $3(5.9)$ & $19(9.0)$ \\
ECMO & $1(0.6)$ & $0(0)$ & $1(2.0)$ & $2(0.9)$ \\
Total & $72(44.7)$ & $8(15.7)$ & $12(23.5)$ & $92(43.4)$ \\
\hline Data presented as n (\%). ECMO, Extracorporeal membrane oxygenation; $N Y H A$, \\
New York Heart Association; $P L E$, protein-losing enteropathy. *Ten patients had un- \\
documented NYHA functional classification, 9 in the open group and 1 in the closed \\
group; for consistency, row percentages calculated using denominator of 161 for open \\
and 51 for closed groups.
\end{tabular}

open group (increased because of skewness in the follow-up interval distribution curve) and 5.0 years (IQR, 3.6-6.9 years) for the closed group.

The only significant difference in outcome measures at the latest follow-up visit was arterial oxygen saturation. In a nonparametric comparison, the closed group had a median oxygen saturation of $96 \%$ (IQR, $94.0 \%-97.0 \%$ ) compared with $91.0 \%$ (IQR, 86.0\%-95.0\%) in the open group $(P<.0001)$.

\section{DISCUSSION}

Our results contribute to the increasing, but incomplete, knowledge pertaining to the management of Fontan fenestrations. The primary finding was that fenestration closure in our cohort was not associated with improved event-free survival, despite a probable influence of selection bias favoring a greater event-free survival in the closed group. Selection bias can be inferred by referring to Table 2-no cases of New York Heart Association class III or IV occurred before fenestration closure in the group that ultimately underwent fenestration closure. In contrast, 27 cases $(16.8 \%)$ of this event occurred in the open group during a comparable follow-up interval. Furthermore, PLE was present in 7 patients in the open group but 0 patients in the closed group. It would be highly provocative to suggest that a patent 4-mm fenestration might cause PLE, although it would be consistent with clinical practice to withhold patients with PLE from referral for fenestration closure.

In general, our results are consistent with a recent report of the Pediatric Heart Network in which no differences were found between groups in terms of thromboembolic events, exercise capacity, ventricular function, peak oxygen consumption, and other measures of performance. ${ }^{6}$ The only 


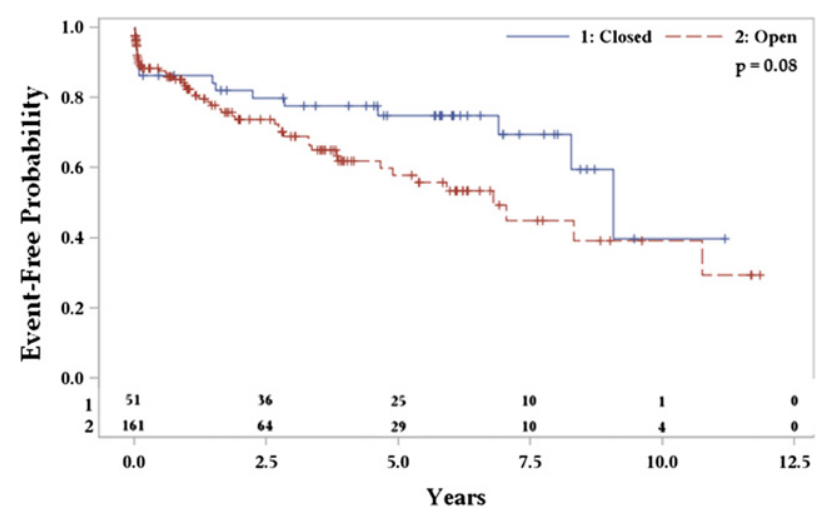

FIGURE 1. Event-free probability curves comparing interval to event in open and closed groups using date of Fontan procedure as time 0 for calculating event intervals.

measure that differed was oxygen saturation, which was 8 points greater in patients with closed fenestrations. Other reports have also shown no difference in maximum oxygen consumption, with patients generally reaching $60 \%$ to $70 \%$ of predicted values, and have highlighted the difficulties in predicting what degree of benefit active fenestration management might provide. ${ }^{6,8-11}$ To date, other than the arterial oxygen saturation, the only documented improvements (in single reports) associated with fenestration closure have been reduced ventilatory abnormalities with exercise and a lower incidence of late tachyarrhythmias. ${ }^{10,12}$ Because most patients in the present report, and in previous reports, had not reached mid-adulthood, the longer term effect of fenestration status on various outcome measures remains unknown.

A second finding of our study was that fenestration closure was associated with a greater follow-up arterial oxygen saturation. This is consistent with expectations and what has been reported by others. ${ }^{6,9,10,13}$ Eliminating cyanosis and potentially at least 1 impetus for ongoing collateral formation and ineffectual cardiac output would seem to be appropriate. However, as noted by others, the long-term clinical relevance of increasing the oxygen saturation from the high 80 s to the mid-90s remains to be verified. ${ }^{6,13}$

A potentially important finding of our study was that median interval to an event was slightly less than the median interval to fenestration closure-events occurred at a median of 1.1 years in the overall cohort and fenestration closure occurred at a median of 1.2 years. This suggests that earlier closure might be necessary to realize any potential benefit. We are aware of 1 previous report by Goff and colleagues, in which fenestration closure occurred at a median of 9 months after the Fontan procedure. ${ }^{13}$ In their study, decompensation after fenestration closure correlated with a shorter interval from the Fontan procedure. ${ }^{13}$ However, their study compared patients before and after fenestration closure and did not

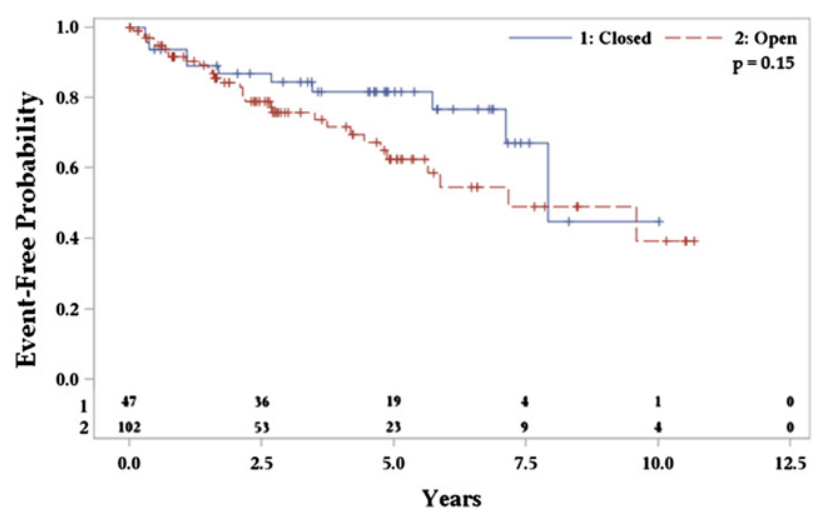

FIGURE 2. Event-free probability curves comparing interval to event in open and closed groups using median interval of fenestration closure as time 0 for calculating event intervals (see text for explanation).

include a comparison group with patent fenestrations. In our opinion, the potential benefit of fenestration closure much earlier in the post-Fontan course remains an unresolved issue.

At present, most available data (although limited) provide no consistent basis for active fenestration closure in all patients. ${ }^{6,8-13}$ Because fenestration closure has yet to demonstrate efficacy for reducing stroke/systemic embolization, the only apparent justification for closure would seem to be clinically significant desaturation, based more so on the secondary effects of cyanosis (not oxygen delivery). ${ }^{6,14,15}$

The important question is whether the quality and magnitude of the available data are sufficient to guide patient treatment. The ongoing variation in management strategies suggests that it is not. From our perspective, 3 options could offer better insight on the issue of optimizing fenestration management. Conducting a randomized controlled trial, although theoretically appealing, would be limited by several practical constraints. A second, and perhaps, more practical option would be modeling the behavior and performance of centers with superior long-term outcomes of single-ventricle management. A third option would be to adopt guidelines from the available evidence that provide a set of management options from which clinicians could agree to choose and consistently follow-this might at least reduce the marked variability in management strategies and enhance the quality of future clinical studies.

\section{Study Limitations}

This was a retrospective study susceptible to various forms of bias. In particular, clinicians might have avoided referring for fenestration closure those patients with more concerning clinical status. An additional limitation was the different follow-up intervals of the 2 groups and the occurrence of several events before fenestration closure in the closed group. Of 
the various methods for addressing this issue, we used the median interval to fenestration closure as the reference point for determining the interval to an event. For both EFP analyses, the length of follow-up was relatively short and precluded evaluation of any potential differences in longer term outcomes. An additional issue was categorizing patients. Given the institutional review board-governed time constraints, certain patients had no accessible follow-up after fenestration closure. We believed the best method for managing this was to categorize such patients in the open group. Also, certain patients underwent attempted fenestration closure but had residual shunts, and certain patients experienced spontaneous closure. It was our best judgment to categorize these patients according to their physiologic status. We performed additional analyses (not presented) with other methods of categorization, and the results were similar. However, we could not be certain that all possible methods of categorization would produce similar results.

\section{CONCLUSIONS}

In the present cohort of fenestrated Fontan patients, fenestration closure was associated with greater arterial oxygen saturation but not greater event-free survival. In the overall cohort, the median interval to an event was somewhat shorter than the median interval to fenestration closure, suggesting potential merit in the evaluation of earlier fenestration closure. Collaborative learning from centers with superior long-term outcomes of single-ventricle management and the adoption of specific fenestration management guidelines might help improve overall outcomes and enhance the quality of future studies.

We acknowledge the financial support of the American Association for Thoracic Surgery's Summer Intern Scholarship.

\section{References}

1. Bridges ND, Lock JE, Castaneda AR. Baffle fenestration with subsequent transcatheter closure: modification of the Fontan operation for patients at increased risk. Circulation. 1990;82:1681-9.

2. Bridges ND, Mayer JE, Lock JE, Jonas RA, Hanley FL, Keane JF, et al. Effect of baffle fenestration on outcome of the modified Fontan operation. Circulation. 1992;86:1762-9.

3. Lemler MS, Scott WA, Leonard SR, Stromberg D, Ramaciotti C. Fenestration improves clinical outcome of the Fontan procedure: a prospective, randomized study. Circulation. 2002;105:207-12.

4. Atz AM, Travison TG, McCrindle BW, Mahony L, Quartermain M, Williams RV, et al. Impact of surgical fenestration in a cohort of Fontan patients [abstract]. J Am Coll Cardiol. 2009;53(suppl 1):A361.

5. Airan B, Sharma R, Choudhary SK, Mohanty SR, Bhan A, Choudhari UK, et al Univentricular repair: is routine fenestration justified? Ann Thorac Surg. 2000; 69:1900-6.

6. Atz AM, Travison TG, McCrindle BW, Mahony L, Quartermain M Williams RV, et al. Late status of Fontan patients with persistent surgical fenestration. J Am Coll Cardiol. 2011;57:2437-43.

7. Tweddell JS, Nersesian M, Mussatto KA, Nugen M, Simpson P, Mitchell ME et al. Fontan palliation in the modern era: factors impacting mortality and morbidity. Ann Thorac Surg. 2009;88:1291-9.

8. Lloyd TR, Rydberg A, Ludomirsky A, Teien DE, Shim D, Beekman RH III, et al. Late fenestration closure in the hypoplastic left heart syndrome: comparison of hemodynamic changes. Am Heart J. 1998;136:302-6.

9. Hijazi ZM, Fahey JT, Kleinman CS, Kopf GS, Hellenbrand WE. Hemodynamic evaluation before and after closure of fenestrated Fontan. An acute study of changes in oxygen delivery. Circulation. 1992;86:196-202.

10. Meadows J, Lang P, Marx G, Rhodes J. Fontan fenestration closure has no acute effect on exercise capacity but improves ventilatory response to exercise. $J \mathrm{Am}$ Coll Cardiol. 2008;52:108-13.

11. Fernandes SM, McElhinney DB, Khairy P, Graham DA, Landzberg MJ, Rhodes J. Serial cardiopulmonary exercise testing in patients with previous Fontan surgery. Pediatr Cardiol. 2010;31:175-80.

12. Ono M, Boethig D, Goerler H, Lange M, Westhoff-Bleck M, Breymann T. Clinical outcome of patients 20 years after Fontan operation-effect of fenestration on late morbidity. Eur J Cardiothorac Surg. 2006;30:923-9.

13. Goff DA, Blume ED, Gauvreau K, Mayer JE Jr, Lock JE, Jenkins KJ. Clinical outcome of fenestrated Fontan patients after closure: the first 10 years. Circulation. 2000;102:2094-9.

14. du Plessis AJ, Chang AC, Wessel DL, Lock JE, Wernosvky G, Newburger JW, et al. Cerebrovascular accidents following the Fontan operation. Pediatr Neurol. 1995;12:230-6.

15. Coon P, Rychik J, Novello RT, Ro PS, Gaynor JW, Spray TL. Thrombus formation after the Fontan operation. Ann Thorac Surg. 2001;71:1990-4. 